\title{
The combination of immunosuppression and carrier cells significantly enhances the efficacy of oncolytic poxvirus in the pre-immunized host
}

\author{
ZS Guo, V Parimi, ME O'Malley, P Thirunavukarasu, M Sathaiah, F Austin and DL Bartlett \\ The University of Pittsburgh Cancer Institute and Department of Surgery, University of Pittsburgh School of Medicine, Pittsburgh, \\ PA, USA
}

\begin{abstract}
Pre-existing antipoxvirus immunity in cancer patients presents a severe barrier to poxvirus-mediated oncolytic virotherapy. We have explored strategies of immunosuppression (IS) and/or immune evasion for efficient delivery of an oncolytic double-deleted vaccinia virus (vvDD) to tumors in the pre-immunized mice. Transient IS using immunosuppressive drugs, including tacrolimus, mycophenolate mofetil and methylprednisolone sodium succinate, have been used successfully in organ transplantation. This drug cocktail alone did not enhance viral recovery from subcutaneous tumor after systemic viral delivery. Using $B$-cell knockout mice, we confirmed that the neutralizing antibodies had a significant role in preventing poxvirus infection. Using a MC38 peritoneal carcinomatosis model,
\end{abstract}

we found that the combination of IS and tumor cells as carriers led to the most effective viral delivery, viral replication and viral spread inside the tumor mass. We found that our immunosuppressive drug cocktail facilitated recruitment of tumor-associated macrophages and conversion into an immunosuppressive M2 phenotype (interleukin (IL)-10 hi/ $\left.\mathrm{IL}-12^{\text {low }}\right)$ in the tumor microenvironment. A combination of IS and carrier cells led to significantly prolonged survival in the tumor model. These results showed the feasibility of treating pre-vaccinated patients with peritoneal carcinomatosis using an oncolytic poxvirus and a combined immune intervention strategy.

Gene Therapy (2010) 17, 1465-1475; doi:10.1038/gt.2010.104; published online 12 August 2010

Keywords: vaccinia virus; pre-immunized host; immunosuppression; carrier cells; tumor-associated macrophages

\section{Introduction}

Oncolytic virotherapy represents a promising, novel approach to cancer treatment. A number of viruses, such as adenovirus, herpes simplex virus, measles virus and vaccinia virus (VACV), are being developed as oncolytic viruses. $^{1-3}$ We and others have been developing VACV and other poxviruses as oncolytic agents. ${ }^{3-12}$ Preclinical studies showed that genetically engineered oncolytic VACV shows both high tumor selectivity and potent antitumoral effects. A phase I clinical trial through intratumoral injection of an oncolytic vaccinia has yielded promising results in patients with hepatocellular carcinoma. ${ }^{13}$ Our genetically engineered virus, called double-deleted vaccinia virus (vvDD), is currently being tested in a phase I clinical trial.

However, despite all impressive progress, the issue of pre-formed immunity has not been adequately addressed. Most cancers occur in older patients who have been vaccinated against smallpox through worldwide smallpox vaccination program, resulting in long-term protection against orthopoxviruses including VACV. Both the neutralizing antibodies and cellular

Correspondence: Dr DL Bartlett or Dr ZS Guo, Department of Surgery, University of Pittsburgh, Pittsburgh, PA 15232, USA.

E-mail: bartlettdl@upmc.edu or guozs@upmc.edu

Received 5 March 2010; revised 15 April 2010; accepted 15 April 2010; published online 12 August 2010 immunity against poxviruses have major roles in protecting the host from infection, and the immunity may last a lifetime. ${ }^{14-17}$ Even in patients who have not been vaccinated against smallpox, antipoxviral immunity will be generated after the initial administration of oncolytic vaccinia. Similar to other anticancer agents, repeated administration of oncolytic viruses will be needed for clinical efficacy. Therefore, it is essential to develop rational strategies that can overcome this hurdle of pre-existing immunity.

It has been observed that vaccinia infection is more severe among people with immunodeficiency diseases and those treated with immunosuppressive medications. ${ }^{18}$ Transient immunosuppression (IS) has been explored as a means of inhibiting immune responses to viruses and virus-induced inflammation in preclinical studies. ${ }^{19}$ Oncolytic virotherapy is enhanced by suppression of both innate and adaptive antiviral responses. ${ }^{20}$ Cyclophosphamide and other immunosuppressive drugs have been used to enhance viral oncolysis and reduce immune components for herpes simplex virus and other oncolytic viruses. ${ }^{20-25}$ However, no previous studies have investigated the effectiveness of immunosuppressive regimens in the context of systemic delivery of oncolytic viruses in animal models with strong pre-existing immunity.

IS has been a standard procedure in organ transplants, ${ }^{26,27}$ and the regimen used successfully for organ transplant might also be useful to inhibit antiviral immunity 
in the setting of oncolytic virotherapy. In this study we investigate multiple immunosuppressive drugs commonly used for inhibition of organ transplant rejection, including tacrolimus (FK-506; Astellas, Pharma US, Inc., Deerfield, IL, USA), mycophenolate mofetil (CellCept; Roche, Nutley, NJ, USA) and methylprednisolone sodium succinate (Solu-Medrol; Pharmacia and Upjohn, New York, NY, USA). FK-506 inhibits calcineurin, inhibiting both T-lymphocyte signal transduction and interleukin-2 (IL-2) transcription, and thus T-cell activation. CellCept depletes guanosine nucleotides preferentially in $\mathrm{T}$ and $\mathrm{B}$ lymphocytes and inhibits their proliferation, thereby suppressing cell-mediated immune responses and antibody formation. ${ }^{28}$ Solu-Medrol is classified as a glucocorticosteroid, an antiinflammatory drug. A combination of these drugs is used clinically and should potently inhibit both cellular immunity and innate immunity. It is important to bear in mind that the tumor microenvironment is progressively immunosuppressive along with tumor development. ${ }^{29,30}$ T-reg cells, myeloid-derived suppressor cells and tumorassociated macrophages (TAMs) are important contributors to the immunosuppressive tumor microenvironment. ${ }^{30-34}$ Dynamic interactions of the tumor microenvironment with oncolytic viruses and/or with immunosuppressive drugs will determine the success of oncolytic virotherapy in the pre-immune host. ${ }^{35-37}$

Autologous carrier cells, as vehicles for delivery of oncolytic viruses, have been investigated in multiple studies using various cell types and viruses. ${ }^{38-40}$ One major advantage has been that the carrier cells 'bypass' the pre-existing humoral immunity against the virus to carry the virus to the tumor tissue. ${ }^{41-44}$ Among a variety of cell types tested, cancer cells have been shown to be effective as carrier cells., ${ }^{2,38-40}$ We hypothesized that either IS with immunosuppressive drugs or immune evasion with carrier cell delivery or the combination of the two would overcome the pre-existing strong antipoxvirus immunity. This combination may allow effective delivery of the vvDD to the tumor, resulting in efficient viral replication and spread and antitumor efficacy in the pre-immunized host.

\section{Results}

$\checkmark v D D$ replicated in tumors in naive mice, but not in the pre-immunized mice

We first tested the possibility of viral replication in the tumor in pre-vaccinated C57BL/6 (B6) mice or naive B6 mice. Naive mice or pre-immunized mice bearing subcutaneous (s.c.) MC38 tumors were treated with vvDD by intraperitoneal (i.p.) injection. Viral recovery from tumor tissues was examined. As shown in Figure $1 \mathrm{a}$, in naive mice, significant amount of vvDD was recovered from tumor tissues on 4 and 8 days after systemic viral administration. Yet, there was no viral recovery from tumors in the pre-immunized mice. High efficiency of replication of vvDD in s.c. and i.p. tumors in naive immunocompetent mice has been shown in previous studies.,10,12 These results confirmed the notion that pre-existing antipoxviral immunity prevented systemic viral delivery and subsequent replication in the tumor tissues.

It has been shown that vaccination with a single dose of highly attenuated vaccinia protect mice with and without immune deficiencies against pathologic vaccinia infection, and the protection comes from both neutralizing antibodies and cellular immunity. ${ }^{17}$ We examined the antipoxvirus neutralizing antibodies in the sera and peritoneal washings from those pre-immunized mice or the mock-vaccinated mice. The antipoxvirus neutralizing antibodies were quantified by a virus neutralization assay on A2780 cancer cells. The concentrations of neutralizing antibodies in the sera from immunized mice were potent, showing 90\% neutralization when diluted tenfold, and over $50 \%$ neutralization even when diluted 200-fold (Figure 1). However, the levels of neutralizing antibodies in the peritoneal washings were below the levels of detection by this assay, partially because of the dilution of peritoneal fluid in washing (data not shown). Nevertheless, our results confirmed that high levels of antipoxvirus neutralizing antibodies were generated in the sera of vaccinated mice. Therefore, some strategies to overcome this antiviral
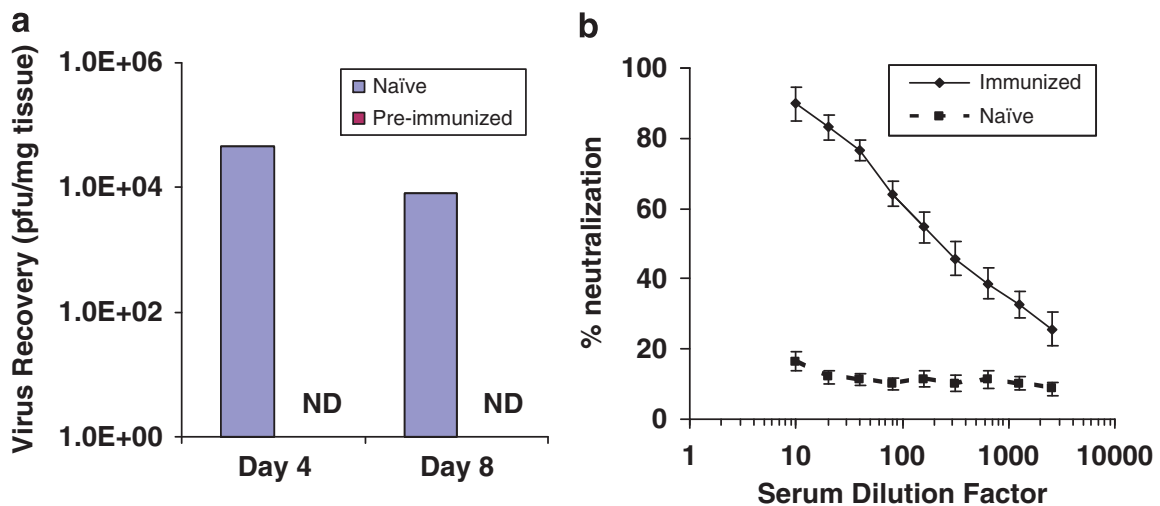

Figure 1 Generation of antipoxviral immunity by vaccination and vaccinia virus replication in tumors of naive, but not pre-immunized B6 mice. (a) Oncolytic virus vvDD was recovered from tumors in naive, but not pre-immunized mice. One group of B6 mice $(n=5)$ was injected i.p. with $4.0 \times 10^{6} \mathrm{pfu}$ of vvDD and housed for 1 month before further experiments. Subcutaneous MC 38 tumors were subsequently established. When tumors reached $\sim 5 \times 5 \mathrm{~mm}$ in size, $1.0 \times 10^{8}$ pfu of vvDD was injected i.p. Tumor tissues were harvested on days 4 and 8 after viral administration, and viral titers were quantified by plaque assays. Data are presented as median values $(P<0.0001)$. ND, no virus detected (in tumors from the pre-immunized mice). (b) Presence of antipoxvirus neutralizing antibodies in the sera from the immunized B6 mice. B6 mice were mock-vaccinated or vaccinated with $4.0 \times 10^{6} \mathrm{pfu}$ of the virus vvDD i.p. After 30 days, sera were collected. vvDD was incubated with the collected sera at the indicated dilutions and the incubated mixtures were used to infect A2780 human cancer cells as described. ${ }^{11}$ Cytopathic effect was observed. Representative data from two experiments are presented as mean \pm s.d. 
Table 1 Doses and actions of the immunosuppressive drugs

\begin{tabular}{|c|c|c|}
\hline Drug & Dosage (1) & Action \\
\hline $\begin{array}{l}\text { Tacrolimus } \\
\text { (Progaf, FK-506) }\end{array}$ & $\begin{array}{l}4 \mathrm{mg} \text { per } \mathrm{kg} \\
\text { body weight } \\
\text { per day }\end{array}$ & $\begin{array}{l}\text { Inhibits calcineurin } \\
\text { and prevents T-cell } \\
\text { activation }\end{array}$ \\
\hline $\begin{array}{l}\text { Mycophenolate } \\
\text { mofetil (CellCept) }\end{array}$ & $\begin{array}{l}80 \mathrm{mg} \text { per } \mathrm{kg} \\
\text { body weight } \\
\text { per day }\end{array}$ & $\begin{array}{l}\text { Inhibits inosine } \\
\text { monophosphate } \\
\text { dehydrogenase and } \\
\text { inhibits } \mathrm{B} \text { and T cells }\end{array}$ \\
\hline $\begin{array}{l}\text { Methylprednisolone } \\
\text { sodium succinate } \\
\text { (Solu-Medrol) }\end{array}$ & $\begin{array}{l}20 \mathrm{mg} \text { per } \mathrm{kg} \\
\text { body weight } \\
\text { per day }\end{array}$ & $\begin{array}{l}\text { As a glucocorticoid and } \\
\text { a general } \\
\text { immunosuppressant. } \\
\text { Exact mechanism of } \\
\text { action unknown }\end{array}$ \\
\hline $\begin{array}{l}\text { Cobra venom } \\
\text { factor }(\mathrm{CVF})\end{array}$ & $\begin{array}{l}500 \mathrm{ng} \mathrm{kg}^{-1} \\
\text { per day }\end{array}$ & $\begin{array}{l}\text { It is the non-toxic, } \\
\text { complement-activating } \\
\text { component of cobra } \\
\text { venom. It causes } \\
\text { depletion of complement }\end{array}$ \\
\hline
\end{tabular}

Drug administration started 2 days before viral challenge and continued daily until the time of killing.

immunity are needed to effectively deliver the virus to the tumor.

\section{Therapeutic levels of immunosuppressive drugs could be achieved after i.p. administration}

We tested the optimal means to deliver the immunosuppressive drugs in mice. We examined the pharmacokinetics of tacrolimus (FK-506), mycophenolate mofetil (CellCept) and methylprednisolone sodium succinate (Solu-Medrol) (Table 1) after different routes of administration: oral, intravenous and i.p. in B6 mice. The concentrations of drugs in the sera at various times after administration were determined. After initial exploration of the three different routes, we chose to deliver the drugs i.p. at the doses of $4 \mathrm{mg} \mathrm{kg}^{-1}$ for FK-506, $20 \mathrm{mg} \mathrm{kg}^{-1}$ for Solu-Medrol and $80 \mathrm{mg} \mathrm{kg}^{-1}$ for CellCept. The concentrations in the sera of the recipient mice held relatively steady at therapeutic levels (FK-506: 4-16 $\mathrm{ng} \mathrm{ml}^{-1}$; CellCept $3-18 \mu \mathrm{g} \mathrm{ml}^{-1}$ ). These results showed that i.p. delivery of the drugs could achieve serum levels that would be therapeutic in human organ transplant.

\section{No productive $V V$ infection in s.c. tumors was achieved after systemic administration in pre-immunized mice under transient IS}

Each of the five individual immunosuppressive drugs and various combinations were applied to the pre-immunized mice bearing s.c. MC38 tumors, followed by i.p. delivery of vvDD. Viral recovery from tumor tissues at 4 and 8 days after viral administration was determined (Table 2). The median values of viral recovery (pfu per $\mathrm{ml}$ ) in all cases were 0 , although there was some viral recovery in a small fraction of mice in some cases. Our results show that cyclophosphamide, FK-506, CellCept, Solu-Medrol, cobra venom factor or combinations of these drugs did not allow productive infection of s.c. tumors in pre-immunized mice. Each of the immunosuppressive drugs target some components of innate and adaptive immunity, but none of them had an effect on the existing circulating neutralizing antibodies, which are a major factor in protecting the host from re-infection by $\mathrm{VACV} \cdot{ }^{14-17}$

\section{Efficient viral recovery was observed only in B-cell knockout $(K O)$ mice in the presence of immunosuppressive drugs}

We then examined whether B cells and neutralizing antibodies were partially responsible for the ineffectiveness of vvDD to reach and replicate in tumor tissues after systemic delivery in the pre-immunized mice. Subcutaneous MC38 tumor-bearing pre-immunized B-cell $\mathrm{KO}$ and wild-type $\mathrm{B} 6$ mice were treated with vvDD in the absence or presence of the immunosuppressive drugs. We then looked for viral gene expression and the recovery of vvDD from MC38 tumor on days 4 and 8 after viral administration (Figure 2). Using vv.luc (luciferase as a viral marker gene) for whole animal live imaging, light was detected in the s.c. MC38 tumor only in the B-cell $\mathrm{KO}$ mice treated with immunosuppressive drugs, indicating active viral transcription in tumor tissue (Figure 2A, image a). Efficient viral recovery of vvDD from MC38 tumor occurred only in the $\mathrm{KO}$ mice with IS on either day 4 or day 8 (Figure 2B). Therefore, activated B cells and the neutralizing antibodies were important components of the barrier to systemic administration of an oncolytic poxvirus in the pre-immunized and tumor-bearing mice.

\section{Cancer cells as carriers did not generate tumor in immunocompetent host}

One of our strategies would be to use MC38 cancer cells as carrier cells to efficiently deliver the oncolytic virus to the i.p. tumor. The safety of using infected tumor cells as carriers was a major concern. Therefore, we tested tumor formation by luciferase-expressing MC38 cancer cells (MC38-luc) either mock-infected or infected with the virus at various multiplicities of infection (MOIs) in syngeneic B6 mice. The formation of i.p. tumor was monitored by assessing luciferase expression through whole-body imaging (Figure 3). By day 7, all mice incubated with mock-infected MC38-luc cells showed significant luciferase expression (Figure 3a). Owing to the overwhelming tumor burden, all mice in this group were killed around day 20, and thus no images from day 36 were available. In contrast, the two groups of mice incubated with MC38-luc cells infected with vvDD at an MOI of either 1 or 10 showed no luciferase expression on day 7 or 36. This indicated that no tumor formation had been observed. In tissue culture, none of MC38-luc cancer cells infected with the virus at an MOI of 1 or 10 survived for more than 3 days (data not shown). Together, these data show that virus-infected MC38 cancer cells were safe as carriers under these conditions in an immunocompetent host.

\section{Strategies to circumvent circulating antibodies were not successful in combination with IS in pre-immunized mice with s.c. tumors}

We explored strategies to circumvent circulating antibodies against vvDD. We examined prolonged IS to target B cells (45 days) to eliminate most of the circulating antibodies. The half-life of circulating antibodies (IgGs) is $\sim 21$ days, and hence prolonged suppression of 
B-cell function should significantly decrease circulating antibodies. We explored the use of anti-IgG therapy before viral infection, and we explored pre-treatment with inactivated vvDD to bind antibodies in advance of live viral delivery. We also examined intravenous delivery of vvDD. In all cases, the median viral recovery

Table 2 Recovery of vvDD from tumors in the pre-immunized B6 mice

\begin{tabular}{lcc}
\hline Drug treatment & $\begin{array}{c}\text { Viral recovery } \\
\text { (median } \\
\text { value) }\end{array}$ & $\begin{array}{c}\text { Animals with low } \\
\text { yet recoverable virus } \\
\text { from tumor (\%) }\end{array}$ \\
\hline CPA & 0 & 0 \\
FK-506 & 0 & 0 \\
CellCept & 0 & 17 \\
Solu-Medrol & 0 & 0 \\
CVF & 0 & 12 \\
Three drugs: CC, SM and FK & 0 & 8 \\
Four drugs: CC, SM, & 0 & 0 \\
FK and CVF & 0 & 40 \\
Three drugs+anti-IgG & 0 & 42 \\
Three drugs for 44 days & 0 & 0 \\
Three drugs+inactivated vvDD & 0 & 20 \\
i.p. carrier cells & &
\end{tabular}

Abbreviations: CC, CellCept; CPA, cyclophosphamide; CVF, cobra venom factor; i.p., intraperitoneal; SM, Solu-Medrol.

All regimens, except that with CPA, were tested in subcutaneous MC38 tumor models in B6 mice $(n=5-12)$. CPA was tested in a peritoneal MC38 tumor model in B6 mice. Viral recovery (pfu per $\mathrm{ml}$ ) was examined in tumor tissues from mice on day 6 or day 8 after viral administration. The three-drug cocktail consisted of FK-506, CellCept and Solu-Medrol, whereas the four-drug cocktail consisted of CVF in addition to the other three drugs. was still zero (Table 2). The highest percentages of tumors with recoverable vvDD, despite low amounts, were in the group receiving 45 days of IS (42\%, in 5 of 12 mice) and those receiving anti-IgG therapy (40\%). Unfortunately, these treatments may not be feasible in cancer patients.

We next examined viral delivery to the tumor in the pre-immunized mice using carrier cells to circumvent the neutralizing antibodies. Infected cells release into the host the extracellular enveloped virus form of VACV, which is resistant to antibody neutralization. ${ }^{45}$ We performed an i.p. injection of carrier cells infected with vvDD at an MOI of 10 in pre-immunized mice bearing s.c. MC38 tumors. We assayed for viral recovery from the tumors in the presence of IS. Again, no significant viral recovery from the tumor was obtained (Table 2).

\section{IS with three drugs and cell carrier delivery of $v V D D$ to pre-immunized mice with peritoneal carcinomatosis (MC38) leads to successful viral recovery}

As the peritoneum seems to lack high levels of antipoxvirus antibodies, we explored the most ideal combination of IS and carrier cell-mediated delivery of vvDD in a peritoneal carcinomatosis model (Figure 4A). Without immunosuppressive drugs and carrier cells, direct delivery of vVDD resulted in no recovery of the virus. With either carrier cells as delivery vehicles (MC38+vvDD) or IS drugs (vvDD/IS) alone, we recovered very low levels of vvDD from tumors-at or below $1.0 \times 10^{2} \mathrm{pfu} \mathrm{mg} \mathrm{m}^{-1}$ on days 4 and 8 . However, when the virus was delivered through carrier cells in the presence of IS, the viral recovery from the tumor reached $5.0 \times 10^{5} \mathrm{pfu} \mathrm{mg}^{-1}$ of protein on day 4 , and still persisted

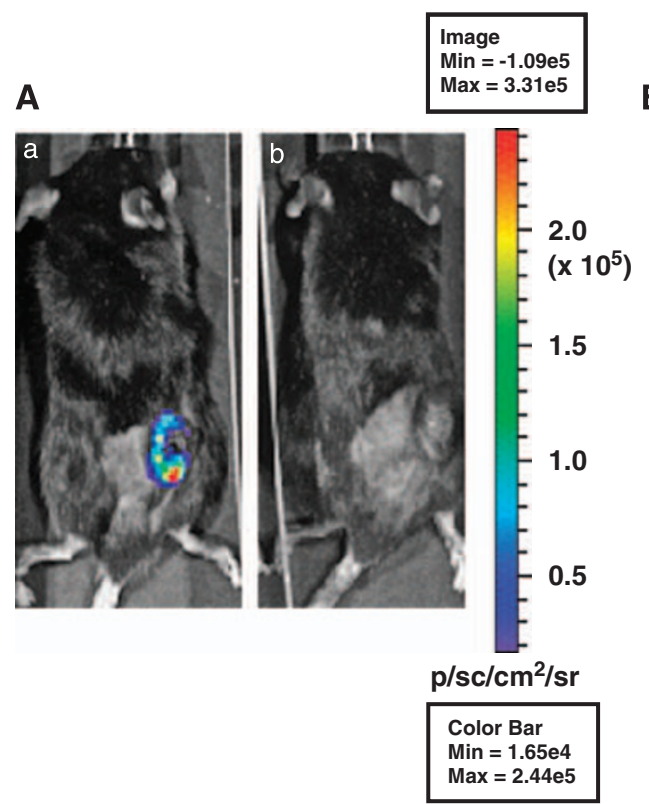

B

\begin{tabular}{|c|c|c|c|}
\hline $\begin{array}{c}\text { Mice } \\
(\mathrm{n}=5)\end{array}$ & $\begin{array}{c}\text { IS } \\
\text { drugs }\end{array}$ & $\begin{array}{c}\text { Harvest time } \\
\text { (days PI) }\end{array}$ & $\begin{array}{c}\text { Viral } \\
\text { yields } \\
\text { (pfu/mI) }\end{array}$ \\
\hline WT & - & 4 & 0 \\
\hline WT & + & 4 & 0 \\
\hline WT & - & 8 & 0 \\
\hline WT & + & 8 & 0 \\
\hline KO & - & 4 & 0 \\
\hline KO & + & 4 & $9.6 \mathrm{E}+06$ \\
\hline KO & - & 8 & 0 \\
\hline KO & + & 8 & $1.4 E+07$ \\
\hline
\end{tabular}

Figure 2 Imaging and viral recovery from tumor in B-cell knockout (KO) mice with or without IS drugs treatment. Viral recovery from tumor tissues in B-cell KO versus wild-type (WT) B6 mice. Mice were vaccinated with $4.0 \times 10^{6}$ pfu per mouse of vvDD i.p. After 30 days (day 0), mice were inoculated with $2 \times 10^{5}$ MC38 cells s.c., and injected with vvDD $\left(5.0 \times 10^{7}\right.$ pfu) i.p. on day 7 with or without the administration of the three IS drug cocktail. (A) Whole animal imaging was conducted on day 4 after viral administration. The intensity of light indicated the level of luciferase expression from virus-infected cells. Typical results of B-cell KO mice with treatment of IS drugs (a) or without IS drugs (b). (B) Tumor tissues were harvested on days 4 and 8 after virus administration ( $n=5)$ and assessed for viral recovery by plaque assays in CV-1 cells. All values are expressed as medians. 
a

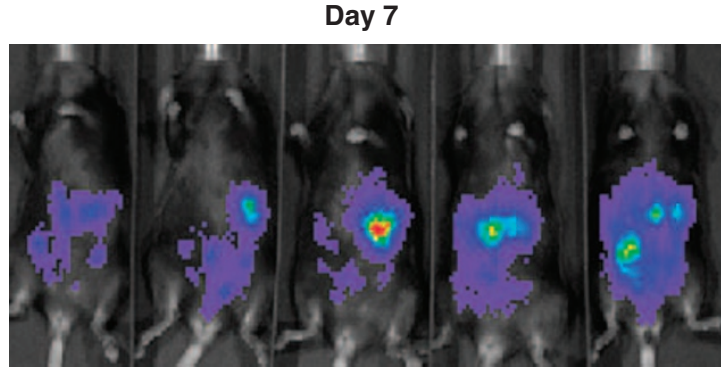

b

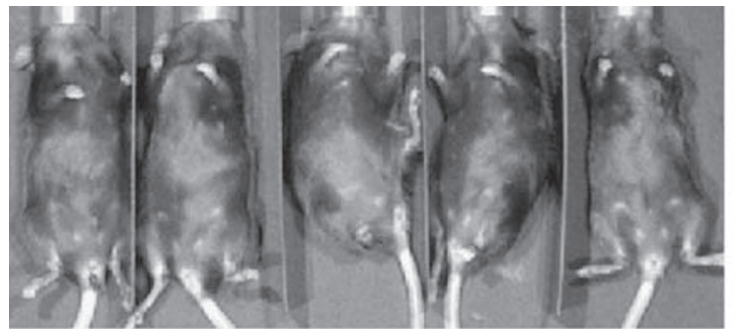

C

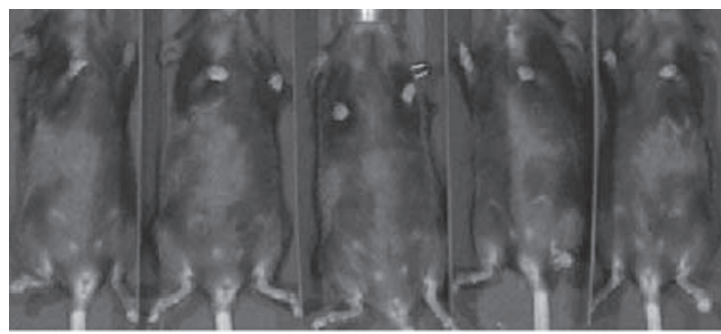

Day 36

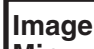

Min $=-6.39 \mathrm{e} 7$

$\operatorname{Max}=5.91 \mathrm{e} 6$

0.8

$\left(\times 10^{8}\right)$

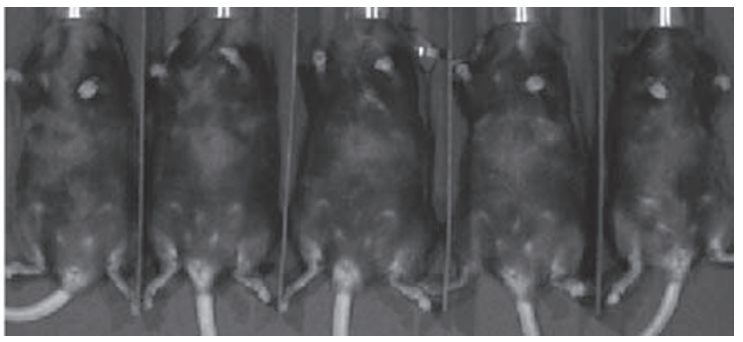

0.6

0.4

0.2

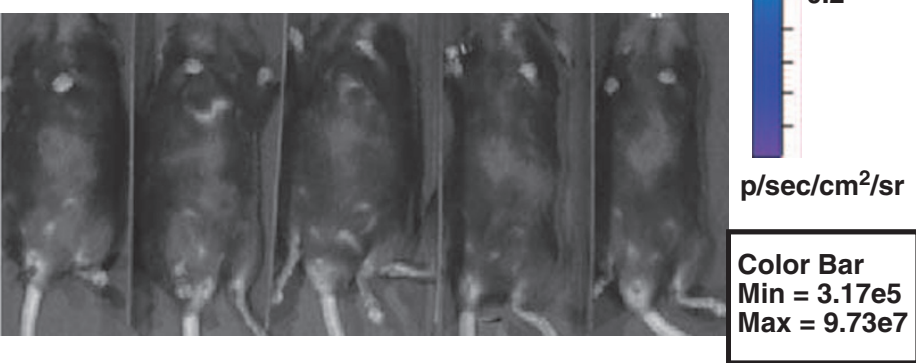

Figure 3 The safety of the virus-infected MC38 cancer cells as carrier cells. The MC38-luc (MC38 cells tagged with the firefly luciferase gene from a lentivirus vector) cancer cells were infected with a vvDD virus at an MOI of 0,1 and 10, as described in the Materials and methods. After infection, $1.0 \times 10^{6}$ MC38-luc cancer cells were injected i.p. into B6 mice. The light images were taken on days 7 and 36 . Mice $(n=5$ per group) were injected with mock-infected MC38-luc cells (a), MC38-luc cells infected with vvDD at an MOI of 1.0 (b) or MC38-luc cells infected with vvDD at an MOI of 10 (c). All mice with mock-infected MC38-luc tumor died around 20 days, and hence no images on day 36 could be obtained.

at $1.0 \times 10^{4} \mathrm{pfu} \mathrm{mg}^{-1}$ on day 8 . The enhanced yield was $>3$ logs over that without IS. Whole animal imaging using vv.luc further confirmed the effectiveness of this approach (Figure 4B). As we see, no luciferase signal was detected from the i.p. tumor in the pre-immunized animal treated with vv.luc alone (Figure $4 B$, panel a). In the presence of IS drugs, but without the use of carrier cells, we detected a weak signal in the tumor area (Figure 4B, panel b). Using both IS drugs and carrier cells, we detected a very robust signal from the tumor tissue (Figure 4B, panel c). These results show that the combination of IS and carrier cells are able to overcome the existing immunity against poxvirus to deliver the virus to a peritoneal tumor.

\section{The immunosuppressive drug combination exerts its functions mainly through M2 TAMs in the tumor microenvironment}

Although some mechanisms of action and side effects of FK506, CellCept and Solu-Medrol have been documented, how they function in combination to provide IS in the tumor microenvironment in the pre-vaccinated host has not been investigated. Three classes of cells, T-reg, TAMs and myeloid-derived suppressor cells, and the immunosuppressive cytokines and chemokines secreted by them, constitute an immunosuppressive tumor microenvironment. ${ }^{29-32}$ To examine the potential contributions of these three types of cells, we divided the pre-immunized, MC38 tumor-bearing mice into two groups: either mock-treated or treated with IS for various durations. We then isolated the infiltrated leukocytes from tumor tissues and identified the cell types by staining with antibodies against cell surface markers of CD4 (CD4 ${ }^{+}$T cells), CD8 (CD8 ${ }^{+}$T cells), CD11c (dendritic cells), NK1.1 (natural killer cells), Ly6G (granulocytes and neutrophils) or Mac-3 (macrophages). MAC-3 is a general marker for macrophages and can be used to distinguish these cells from lymphocytes. The stained cells were then analyzed by flow cytometry.

Few $\mathrm{CD}^{+}$and $\mathrm{CD}^{+} \mathrm{T}$ cells, $\mathrm{CD}^{+} 1 \mathrm{c}^{+}$dendritic cells or $\mathrm{NK} 1.1^{+}$natural killer cells infiltrated into the tumor tissue in pre-immunized mice in the absence or presence of IS (Figure 5a). These results effectively prevented us from further meaningful analysis of the effect of T-reg cells on this regimen. However, these results were not unexpected because FK-506 and CellCept together inhibit the proliferation, differentiation and function of $\mathrm{T}$ cells, including T-reg cells. As for myeloid-derived suppressor cells, we observed a tendency, although 
A

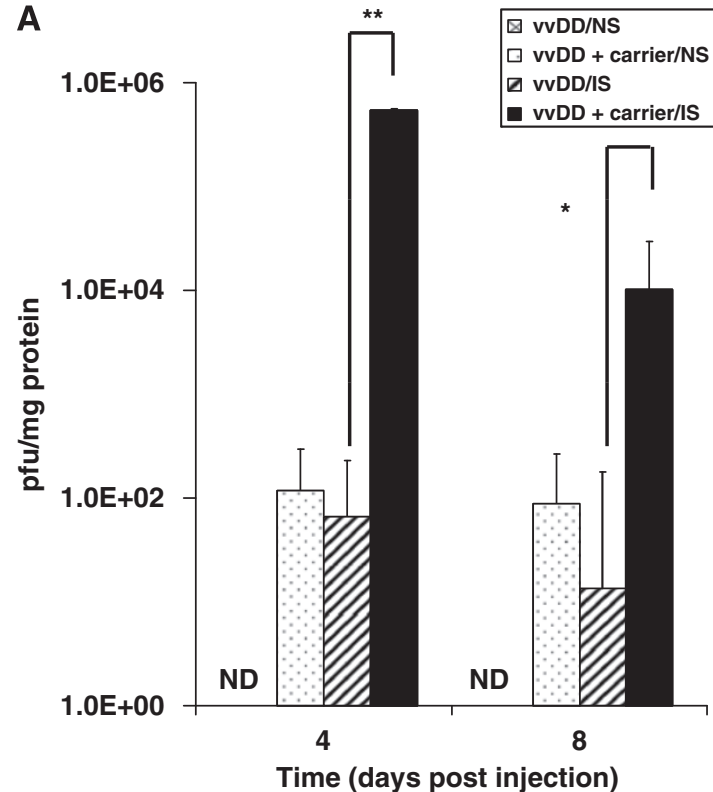

(days post injection)
B

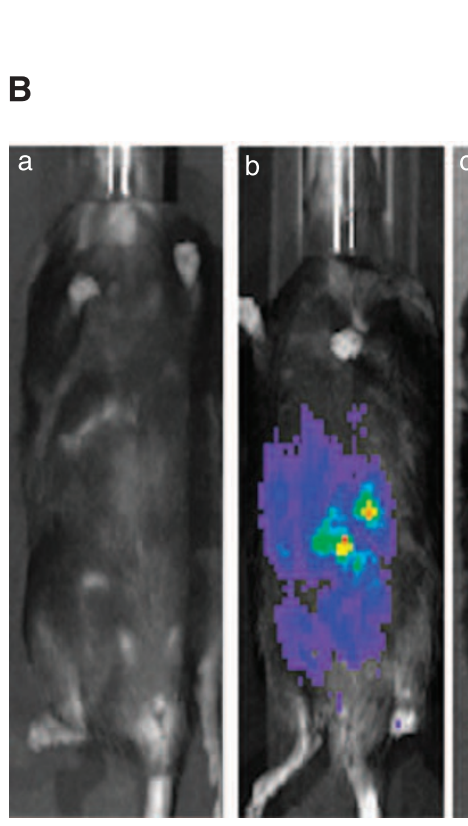

Image

Min $=-1.08 \mathrm{e} 5$

$\operatorname{Max}=2.17 \mathrm{e} 5$

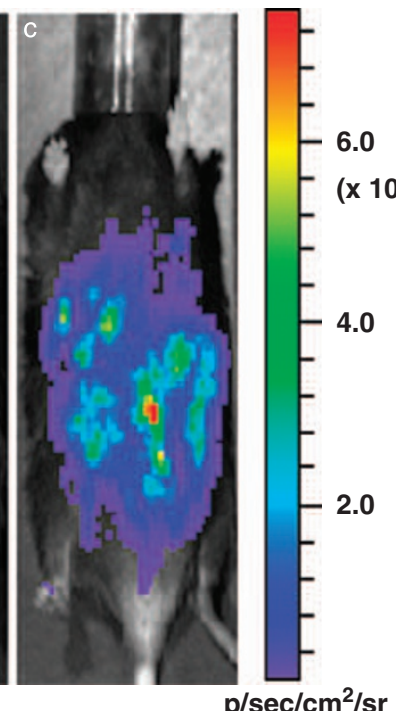

$\mathrm{p} / \mathrm{sec} / \mathrm{cm}^{2} / \mathrm{sr}$

Color Bar

Min $=7.46 \mathrm{e} 3$

$\operatorname{Max}=7.46 \mathrm{e} 5$

Figure 4 The combined effects of IS drugs and carrier cells on viral replication and viral gene expression (firefly luciferase as viral marker gene) in mice bearing intraperitoneal (i.p.) MC38 colorectal carcinomatosis. B6 mice were pre-vaccinated with vvDD at $4.0 \mathrm{E} 6$ pfu per mouse on day -30 . The mice were inoculated with MC38 cancer cells $\left(2 \times 10^{5}\right)$ i.p. (as day 0$)$. On day 7 , mice were treated i.p. with either vvDD alone (5.0E7 pfu per mouse) or with 5.0E6 MC38 cells infected ex vivo with vvDD at an MOI of 5.0, as described in the Materials and methods ( $n=15$ per group). (A) The viral recovery from cancer cells in the peritoneal cavity at days 4 and 8 after viral delivery. IS, animals with IS; ND, not detected; NS, non-immunosuppressed. ${ }^{*} P<0.032 ;{ }^{*} P<0.05$. (B) Live whole animal imaging after virus delivery. The pre-immunized and tumor-bearing mice were injected i.p. with $1.0 \mathrm{E} 8 \mathrm{pfu}$ of vv.luc per mouse. The imaging was performed on day 4 after viral injection as described. ${ }^{11}$ The representative images were from mice with MC38 i.p. tumors with and treated with either vv.luc alone (a), IS and vv.luc (b) or IS and vv.luc delivered through MC38 carrier cells (c).

statistically insignificant, of more $\mathrm{Ly} 6 \mathrm{G}^{+}$cells (mostly granulocytes and neutrophils) in the tumors in the group treated with IS (6.25 versus $1.63 \%$ for IS versus mocktreated mice; $P=0.109$ ).

The most striking results came from MAC- $3^{+}$cells that are potentially immunosuppressive TAMs. We observed a marked increase of $\mathrm{MAC}-3^{+}$cells in the tumors in the IS-treated mice (26.86 versus $4.5 \%$ for IS versus mocktreated mice; $P=0.030$ ). TAMs with M2 phenotype have been shown to be highly immunosuppressive. ${ }^{27,30}$ In contrast, the M1 macrophages are immunoactive. Two cytokines, IL-10 and IL-12, are key markers of M1 (IL-10 $0^{\text {low }} /$ IL- $12^{\text {hi }}$ ) or M2 (IL-10 $10^{\text {hi }} /$ IL- $12^{\text {low }}$ ) macrophages. TAMs were isolated from the tumors in animals mocktreated or treated with IS. Peritoneal macrophages (pMACs) were isolated from naive B6 mice and served as controls. Real-time reverse transcriptase-PCR was used to quantify the relative levels of mRNAs encoding these two cytokines. Previously, F4/80+ TAMs have been well characterized. ${ }^{46}$ Therefore, we isolated the $\mathrm{F} 4 / 80^{+}$/ Mac- $3^{+}$dual positive TAMs from groups of mice mocktreated or treated with IS drugs by flow cytometry (Figure 5b). The levels of mRNA encoding IL-10 and IL-12p40 were analyzed by quantitative real-time reverse transcriptase PCR, normalized to the level of GAPDH mRNA (Figure 5c). In control naive pMAC (pMAC-N) and activated pMAC (pMAC-A), IL-10 mRNA was low.
In TAMs isolated from mock-treated mice (TAMs-NS), IL-10 was also low. However, IL-10 mRNA in IS-treated group (TAM-IS) increased approximately sixfold. The expression patterns of IL- $12 b$ in pMAC-N and pMAC-A were as expected, with high level in activated pMACs, representing the M1 phenotype. The levels of IL-12b were low in both TAM-NS and TAM-IS. These results showed that TAMs from the IS-treated mice, but not control mice, were markedly increased in number and showed a typical immunosuppressive M2 phenotype.

The combination of IS and cell carrier delivery leads to an enhanced therapeutic effect and prolonged survival in mice with peritoneal carcinomatosis

We then tested the efficacy of vvDD using the combined approach of IS and cell carrier delivery to MC38 peritoneal carcinomatosis in the pre-immunized mice. We had first evaluated the effects of immunosuppressive drugs on tumor growth and survival. In repeated experiments, we did not find significant difference in survival in tumor-bearing mice with or without transient IS (data not shown). Therefore, in subsequent experiments, all mice were subject to IS and then underwent subsequent treatment with either Hank's balanced salt solution saline, vvDD (naked) or vvDD delivered by carrier cells (Figure 6). In this tumor model with IS, the 

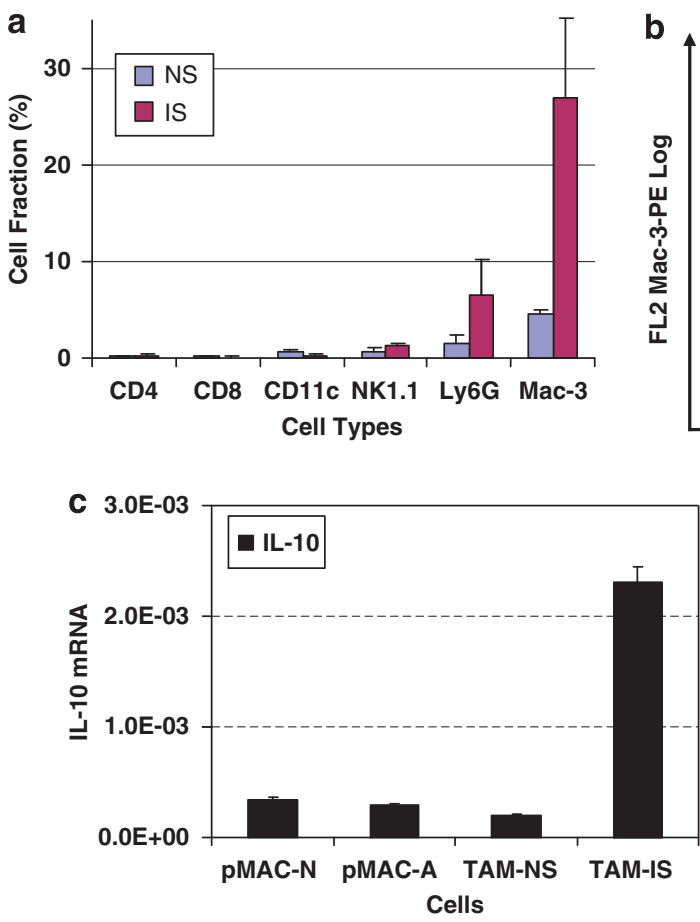

b

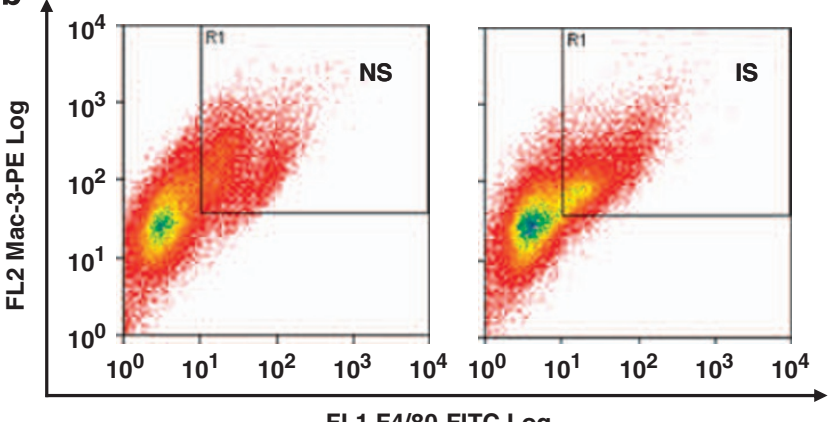

FL1 F4/80-FITC Log

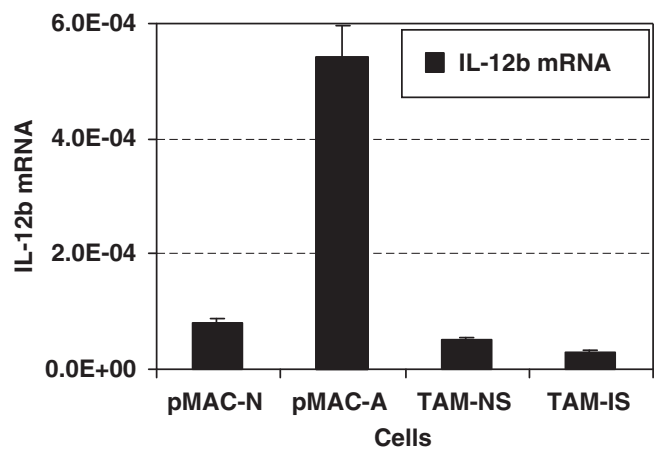

Figure 5 Analyses of the tumor-infiltrated leukocytes in the tumor of pre-immunized hosts mock-treated or treated with immunosuppressive drugs on day 4 after viral administration. (a) Relative quantities of major classes of leukocytes as analyzed by immunostaining of cell surface markers and then flow cytometry. The antibodies are against cell surface proteins of CD4 (CD4 ${ }^{+} \mathrm{T}$ cells), CD8 (CD8 ${ }^{+}$T cells), CD11c (dendritic cells), NK1.1 (natural killer (NK) cells), Ly6G (granulocytes and neutrophils) and MAC-3 (macrophages). (b) Examples of flow cytometric analysis of $\mathrm{F} 4 / 80^{+} / \mathrm{MAC}-3^{+}$dual positive TAMs in the tumors of pre-immunized mice with or without treatment with IS drugs. (c) Expression of two key cytokines in F4/80+/MAC-3+ dual positive TAMs from tumors in either IS drug-treated or untreated mice, and naive and activated pMACs serve as controls. Total RNAs were purified by standard procedure as described. The expression of murine IL-10 and IL-12/P40 mRNA (left and right graphs) was quantified by real-time reverse transcriptase PCR (RT-PCR). The copy number of target mRNA was normalized to that of the housekeeping gene GAPDH. Data represent mean \pm s.e.m.

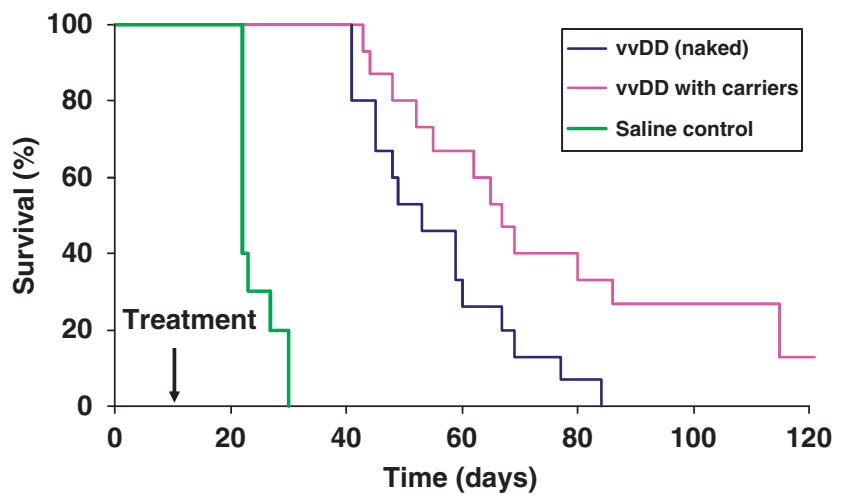

Figure 6 The combined effects of immunosuppressive drugs and carrier cells on the efficacy of vvDD oncolytic virotherapy in preimmunized mice bearing intraperitoneal (i.p.) MC38 colorectal carcinomatosis. B6 mice were pre-immunized for 1 month before inoculation of MC38 tumor cells for i.p. colorectal carcinomatosis. At 10 days after tumor cell inoculation, IS was initiated in all mice ( $n=15$ per group), and then treated with either Hank's balanced salt solution (HBSS) saline (CTL), vvDD (vvDD naked) or vvDD delivered through MC38 carrier cells (vvDD with carriers). The survival and health of these mice were closely monitored. Survival data were plotted on a Kaplan-Maier curve. Statistical comparisons were performed using a log-rank test. $P<0.0001$ (control vs naked); $P<0.00001$ (control vs vvDD with carriers); $P \leqslant 0.017$ (vvDD (naked) vs vvDD with carriers). saline-treated mice became moribund around day 21 and all required euthanasia by day 31, with a median survival of 22 days (control group). However, in animals treated with vvDD (naked), the median survival extended to 52 days $(P<0.0001)$. The addition of carrier cells for delivery of vvDD further enhanced the survival duration, with a median of 67 days $(P \leqslant 0.017$ compared with vvDD naked; $P<0.00001$ compared with salinetreated group). These results showed that combined IS and immunoevasion is effective in overcoming pre-existing antipoxvirus immunity to successfully deliver the virus regionally to the tumor in the peritoneal cavity, leading to successful oncolytic virotherapy in the pre-immunized host.

\section{Discussion}

Two major issues prompted us to study the use of immunosuppressive drugs for oncolytic poxvirus therapy in the pre-immunized host. First, pre-existing antipoxvirus immunity in most cancer patients has presented a barrier to the use of oncolytic poxviruses, especially for systemic treatment. Second, successful therapy using oncolytic poxviruses may require repeated administration of poxviruses to the same patients in 
whom the antipoxvirus immunity is generated de novo or boosted after the initial dose of an oncolytic poxvirus. Therefore, to fully realize the potential of oncolytic poxviruses, rational strategies to effectively evade and/ or suppress the pre-existing antipoxvirus immunity must be investigated.

In this study, we have investigated the strategies of IS, IE or the combination to bypass the antipoxvirus immunity and effectively deliver a poxvirus to tumor models by regional or systemic delivery. As a pre-clinical project, we were interested in testing approved drugs and combinations that have been used frequently in clinical practice in the setting of transplantation. Initially, we tested individual immunosuppressive drugs and then various combinations. Immunosuppressive drugs alone did not allow for systemic delivery of vvDD to s.c. tumors. In humans, it has been shown that the neutralizing antibodies produced by smallpox vaccination were a key component to protection from smallpox infection. ${ }^{14,16}$ The antipoxvirus neutralizing antibodies were presumed to be the main barrier to successful administration of the oncolytic vvDD to tumors in the pre-immunized mice. Our results from B-cell $\mathrm{KO}$ mice confirmed that notion. The absence of $\mathrm{B}$ cells alone would not allow successful delivery of the virus, but the combination of IS and the absence of B cells led to efficient delivery of VvDD to the tumors.

Many different strategies to overcome circulating antibodies have been investigated, including anti-IgG therapy, decoy antigens, prolonged IS and plasmapheresis. We explored many of these without success. Other investigators have previously shown that carrier cells are able to systemically deliver oncolytic viruses to tumor even in the presence of neutralizing antibodies against particular viruses such as adenovirus or measles virus. ${ }^{41-44,47}$ The effectiveness of a particular cell-based system of oncolytic virus delivery rests on three sequential phases: ex vivo loading, in vivo targeting and virus production at the tumor site. ${ }^{39}$ We chose to use cancer cells as carrier cells as they are easy to obtain in abundance, and they show high productivity of the progeny viruses. ${ }^{39,40}$ Unfortunately, the use of cancer cells as delivery vehicles was still not effective at allowing vvDD infection of s.c. tumors, even in the setting of IS. It is likely that trafficking of the carrier cells to the s.c. tumor was poor. Although numerous strategies to potentially improve trafficking and for overcoming circulating antibodies should be explored in more detail, we chose to study i.p. delivery of vvDD in a model of peritoneal carcinomatosis, in which targeting is less of an issue. We found that only the combination of carrier cells and IS led to efficient viral recovery from the peritoneal tumor. This combination, however, was effective enough to lead to a threefold increase in survival duration compared with control mice.

Immunosuppressive drugs may exert their effects on oncolytic viruses by multiple mechanisms. First, they may inhibit proliferation and activities of immune cells in both adaptive and innate immunity arms. By doing so, they may inhibit the antiviral inflammatory responses that lead to swift viral clearance. ${ }^{36,48,49}$ Mononuclear cells, including natural killer cells and microglia/macrophages, are involved in the clearance of herpes simplex virus, and cyclophosphamide pretreatment inhibited herpes simplex virus-induced infiltration of tumor-associated phagocytic cells. ${ }^{25}$ Our current study revealed that a cocktail of three drugs helped in both recruitment and education of the infiltrated monocytes into M2-polarized, immunosuppressive TAMs. In addition, the quantity of $\mathrm{Ly}_{6 \mathrm{G}}{ }^{+}$cells, some of which are myeloid-derived suppressor cells, was also enhanced in the tumors in the IS-treated mice. These effects may lead to prolonged virus survival in the tumor microenvironment and enhanced therapeutic efficacy.

It is interesting to note that innate immunity and inflammatory responses to oncolytic virus can serve as a double-edged sword. On one hand, it will promote the antiviral clearance prematurely and thus reduce the efficacy of the virus, and on the other, the inflammatory response to the virus may lead to bystander killing of uninfected cancer cells and long-lasting antitumor immunity. ${ }^{35-37}$ In our case, IS promotes the education of TAMs into highly immunosuppressive M2 phenotype, which may promote a friendly environment for replication and spread of an oncolytic poxvirus. At the same time, these M2-type TAMs may promote tumor angiogenesis, tumor cell invasion and matrix remodeling. ${ }^{32-34,46}$ Oncolytic VACV has repeatedly been shown to be more efficacious in nude mice than immunocompetent mice, suggesting that direct viral oncolysis is the most significant contributor to its overall effect. In this regard, IS may be more beneficial than detrimental in the setting of oncolytic poxvirus.

Any IS required for cancer treatment will be transient, and the recovering immune response to the virus may lead to efficient bystander immune clearance of cancer cells and long-term immune memory. Although beyond the scope of this study, the time course and effect of immune recovery in this context needs further study.

The utility of cancer cells as carriers for oncolytic viruses has raised safety issues. ${ }^{40,47}$ Additional safeguards will need to be implemented against those cancer cells that might escape infection and killing by the oncolytic virus. These strategies include irradiating the cancer cells before administration, or engineering the virus with a suicide gene system. ${ }^{40,47}$ We have developed another version of our virus, vvDD-CD, ${ }^{4}$ expressing the yeast cytosine deaminase that would provide an effective suicide enzyme system to kill infected cells and neighboring uninfected cancer cells through a bystander effect. The safety and effectiveness of suicide gene-armed virus-infected cancer cells as carriers will be examined in the future.

\section{Materials and methods}

\section{Cell lines}

MC38 and MOSEC murine cancer cells, A2780 and HeLa human cancer cells, and monkey kidney CV-1 cells have been used in our previous studies. .,10,12 $^{-}$

\section{Vaccinia virus}

All VACVs were derived from Western Reserve strain. The virus vvDD-EGFP (vvDD in short), with dual deletions of viral genes $t k$ and $v g f$, has been characterized previously. $^{9}$ The pseudo-wild-type virus vF13L, and vv.luc, which expresses firefly luciferase, have also been described. ${ }^{9,11}$ 


\section{Mice and experimental procedures}

Female C57BL/6 mice (B6) were purchased from Taconic Farms, Inc. (Germantown, NY, USA). The B-cell KO C57BL/6 mice, B6.129S2-Igh- $6^{t m 1 C g n} / \mathrm{J}$, were purchased from Jackson Laboratory (Bar Harbor, ME, USA).

The animal studies were approved by the institutional animal care and use committee of the University of Pittsburgh. Mice were housed in standard conditions and given food and water ad libitum.

\section{Pre-immunization with vVDD and assay for neutralizing antibody}

The B6 mice were injected i.p. with vvDD at $4.0 \times 10^{6} \mathrm{pfu}$ per mouse. After 1 month, they were used for tumor cell inoculation and additional experiments.

For determination of the titer of antipoxvirus neutralizing antibodies in the mouse serum, antisera samples were diluted to appropriate concentrations, and incubated with vvDD, and then were used to infect A2780 human cancer cells. The cytopathic effect was determined as described. ${ }^{11,17}$

\section{Administration of the IS drugs into mice and quantification for serum IS drugs}

Immunosuppressive drugs tacrolimus (FK-506; fujimycin), mycophenolate mofetil (CellCept), methylprednisolone sodium succinate (Solu-Medrol), and agent cobra venom factor have been tested in this study. The prescription drugs Prograf (tacrolimus; Astellas), CellCept Intravenous (Roche) and Solu-Medrol (Pharmacia and Upjohn) were obtained from the Hillman Cancer Center Pharmacy (Pittsburgh, PA, USA). CellCept in powder form was dissolved in 5\% dextrose injection USP (D5W) to desirable concentration. FK-506 in solution and methyl prednisolone powder were diluted or dissolved in $0.9 \%$ normal saline to desirable concentration.

Initially, immunosuppressive drug cocktail was given in oral, intravenous and i.p. routes. The concentrations of drugs in the serum were tested at various time points after administration. After initial investigation, we chose the delivery of the drugs by i.p. route for all the remaining experiments. All the three drugs were mixed together and injected i.p. in a total solution of $200 \mu \mathrm{l}$ per day in a single dose. Daily injection site was altered to minimize local fibrosis and infection. The drugs are titrated to 1-month-old mice based on total body weight. On the basis of initial pilot experiments, the weight-adjusted doses of IS drugs were derived by measuring serum concentration at 2 to $48 \mathrm{~h}$. Corresponding human weight-adjusted doses were used as comparative standard. The mouse serum drug trough levels were always higher than the recommended serum concentration in human organ transplant patients.

The concentrations of the drugs in the sera from mice were determined by the clinical laboratory at the University of Pittsburgh Medical Center.

\section{Tumor models and antitumor effects}

In the first tumor model, $2.0 \times 10^{5} \mathrm{MC} 38$ colon cancer cells in $100 \mu \mathrm{l}$ of Dulbecco's modied Eagle's medium were injected s.c. into the right flanks of 7-week-old female nude mice and allowed to grow for 7-10 days. In the second model, $2.0 \times 10^{5} \mathrm{MC} 38$ colon cancer cells were injected into the peritoneal cavity as colorectal carcinomatosis model. ${ }^{4,12}$

\section{Cancer cells as carrier cells}

MC38 cancer cells were infected with vvDD at an MOI of 10 . They were harvested at $16 \mathrm{~h}$ after infection. The infected cells were harvested, washed in cold $1 \times$ phosphate-buffered saline thrice, counted and injected i.p. into recipient mice. The viability of the infected cancer cells were monitored by Trypan blue exclusion staining at the time of harvest and afterward in vitro.

RNA isolation and real-time reverse transcriptase $P C R$ The procedure of total RNA purification, real-time reverse transcriptase PCR was performed as described previously..$^{50}$ In brief, total RNA was extracted from cells using RNeasy mini kit (Qiagen, Valencia, CA, USA). First-strand complementary DNA was synthesized using ImProm-II reverse transcription system with an oligo $(\mathrm{dT})_{15}$ primer (Promega, Madison, WI, USA). Real-time PCR was performed on complementary DNA using TaqMan gene expression assays specific for murine IL-10 (Mm99999062_m1), IL-12p40 (Mm99999067_m1) and Gapdh (Mm99999915_m1), with an ABI StepOnePlus Real-Time PCR System (Āpplied Biosystems, Foster City, CA, USA).

\section{Live whole animal imaging}

The in vivo optical imaging for the animals was performed using a Xenogen IVIS 200 Optical In Vivo Imaging System (Caliper Life Sciences, Hopkinton, MA, USA), with technical assistance from the Small Animal Imaging Core Facility of the University of Pittsburgh Cancer Institute.

\section{Isolation of tumor-infiltrated leukocytes and flow cytometry}

The isolated leukocytes were probed with fluorescein isothiocyanate (FITC) rat anti-mouse CD4, FITC rat anti-mouse CD6, FITC hamster anti-mouse CD11c, phycoerythrin (PE) mouse anti-mouse NK-1.1, PE rat anti-mouse Ly-6G and Ly-6C or PE rat anti-mouse MAC-3 antibody, or isotype Ig controls (BD Pharmingen Inc., San Diego, CA, USA). The stained cells were subject to flow cytometry. For isolation of $\mathrm{F} 4 / 80^{+} / \mathrm{MAC}-3^{+}$ dual positive TAMs, cells were probed with both PE-rat anti-MAC-3 antibody (BD Pharmingen) and FITC-rat anti-mouse F4/80 antibody (BioLegend, San Diego, CA). The dual positive cells were sorted using a MoFlo cell sorter (Beckman Coulter, Fort Collins, CO, USA). Data were analyzed using the software Summit version 4.3 (Beckman Coulter, Inc., Brea, CA, USA).

\section{Isolation and activation of peritoneal macrophages}

We have followed a standard procedure for isolation of murine pMACs and activation of these cells in vitro. ${ }^{51,52}$ In brief, naive B6 mice were injected peritoneally with $3.0 \%$ thioglycollate medium (Fisher Scientific, Pittsburgh, PA, USA). After 4 days, mice were injected i.p. with $5 \mathrm{ml}$ of ice-cold medium with 5\% de-complemented fetal bovine serum, and the peritoneal washes were collected. Cells were plated on tissue culture plates for $1 \mathrm{~h}$, and then non-adherent cells were aspirated. The adherent cells were washed twice with $1 \times$ phosphate-buffered 
saline before fresh growth medium was added. The purity of macrophages isolated by this protocol was $>90 \%$. For activated macrophages, the cells were treated first with $150 \mathrm{U} \mathrm{ml}^{-1}$ murine interferon- $\beta$ (Peprotech, Rocky Hill, NJ, USA) for $12 \mathrm{~h}$, and then with $10 \mathrm{ng} \mathrm{ml}^{-1}$ lipopolysaccharides (Sigma-Aldrich, St Louis, MO, USA) for $18 \mathrm{~h}$.

\section{Statistics}

The statistical analyses were performed as described previously. ${ }^{4,10}$ The $P$-value of $<0.05$ was considered statistically significant.

\section{Conflict of interest}

DLB is a consultant of the Jennerex BioTherapeutics, a company developing oncolytic viruses.

\section{Acknowledgements}

We thank Noriko Murase and Venkat Venkataramanan at the University of Pittsburgh for their initial expert advice on immunosuppressive drugs. The imaging technical services were provided by the Small Animal Imaging Core Facility at the UPCI. We also thank the Flow Cytometry Core at UPCI for the technical help in flow cytometry. This study was supported in part by the NIH grants R01-CA100415 and P01-CA132714, and by The David C Koch Regional Therapy Cancer Center.

\section{References}

1 Parato KA, Senger D, Forsyth PA, Bell JC. Recent progress in the battle between oncolytic viruses and tumours. Nat Rev Cancer 2005; 5: 965-976.

2 Guo ZS, Thorne SH, Bartlett DL. Oncolytic virotherapy: molecular targets in tumor-selective replication and carrier cell-mediated delivery of oncolytic viruses. Biochim Biophys Acta 2008; 1785: 217-231.

3 Kirn DH, Thorne SH. Targeted and armed oncolytic poxviruses: a novel multi-mechanistic therapeutic class for cancer. Nat Rev Cancer 2009; 9: 64-71.

4 Chalikonda S, Kivlen MH, O'Malley ME, Eric Dong XD, McCart JA, Gorry MC et al. Oncolytic virotherapy for ovarian carcinomatosis using a replication-selective vaccinia virus armed with a yeast cytosine deaminase gene. Cancer Gene Ther 2008; 15: 115-125.

5 Zhang Q, Yu YA, Wang E, Chen N, Danner RL, Munson PJ et al. Eradication of solid human breast tumors in nude mice with an intravenously injected light-emitting oncolytic vaccinia virus. Cancer Res 2007; 67: 10038-10046.

6 Tysome JR, Briat A, Alusi G, Cao F, Gao D, Yu J et al. Lister strain of vaccinia virus armed with endostatin-angiostatin fusion gene as a novel therapeutic agent for human pancreatic cancer. Gene Therapy 2009; 16: 1223-1233.

7 Guse K, Sloniecka M, Diaconu I, Ottolino-Perry K, Tang N, $\mathrm{Ng} \mathrm{C}$ et al. Antiangiogenic arming of an oncolytic vaccinia virus enhances antitumor efficacy in renal cell cancer models. J Virol 2010; 84: 856-866.

8 Yu Z, Li S, Brader P, Chen N, Yu YA, Zhang Q et al. Oncolytic vaccinia therapy of squamous cell carcinoma. Mol Cancer 2009; 8: 45 .

9 McCart JA, Ward JM, Lee J, Hu Y, Alexander HR, Libutti SK et al. Systemic cancer therapy with a tumor-selective vaccinia virus mutant lacking thymidine kinase and vaccinia growth factor genes. Cancer Res 2001; 61: 8751-8757.

10 Guo ZS, Naik A, O'Malley ME, Popovic P, Demarco R, Hu Y et al. The enhanced tumor selectivity of an oncolytic vaccinia lacking the host range and antiapoptosis genes SPI-1 and SPI-2. Cancer Res 2005; 65: 9991-9998.

11 Thorne SH, Hwang TH, O'Gorman WE, Bartlett DL, Sei S, Kanji $\mathrm{F}$ et al. Rational strain selection and engineering creates a broad-spectrum, systemically effective oncolytic poxvirus, JX-963. J Clin Invest 2007; 117: 3350-3358.

12 Yang S, Guo ZS, O'Malley ME, Yin X, Zeh HJ, Bartlett DL. A new recombinant vaccinia with targeted deletion of three viral genes: its safety and efficacy as an oncolytic virus. Gene Therapy 2007; 14: 638-647.

13 Liu TC, Hwang T, Park BH, Bell J, Kirn DH. The targeted oncolytic poxvirus JX-594 demonstrates antitumoral, antivascular, and anti-HBV activities in patients with hepatocellular carcinoma. Mol Ther 2008; 16: 1637-1642.

14 Amanna IJ, Slifka MK, Crotty S. Immunity and immunological memory following smallpox vaccination. Immunol Rev 2006; 211: 320-337.

15 Crotty S, Felgner P, Davies H, Glidewell J, Villarreal L, Ahmed R. Cutting edge: long-term $\mathrm{B}$ cell memory in humans after smallpox vaccination. J Immunol 2003; 171: 4969-4973.

16 Hammarlund E, Lewis MW, Hansen SG, Strelow LI, Nelson JA, Sexton GJ et al. Duration of antiviral immunity after smallpox vaccination. Nat Med 2003; 9: 1131-1137.

17 Wyatt LS, Earl PL, Eller LA, Moss B. Highly attenuated smallpox vaccine protects mice with and without immune deficiencies against pathogenic vaccinia virus challenge. Proc Natl Acad Sci USA 2004; 101: 4590-4595.

18 CDC. Recommendations of the Advisory Committee on Immunization Practices (ACIP): use of vaccines and immune globulins for persons with altered immunocompetence. MMWR Recomm Rep 1993; 42: 1-18.

19 Arruda VR, Favaro P, Finn JD. Strategies to modulate immune responses: a new frontier for gene therapy. Mol Ther 2009; 17: 1492-1503.

20 Ikeda K, Ichikawa T, Wakimoto H, Silver JS, Deisboeck TS, Finkelstein D et al. Oncolytic virus therapy of multiple tumors in the brain requires suppression of innate and elicited antiviral responses. Nat Med 1999; 5: 881-887.

21 Thomas MA, Spencer JF, Toth K, Sagartz JE, Phillips NJ, Wold WS. Immunosuppression enhances oncolytic adenovirus replication and antitumor efficacy in the Syrian hamster model. Mol Ther 2008; 16: 1665-1673.

22 Hirasawa K, Nishikawa SG, Norman KL, Coffey MC, Thompson BG, Yoon CS et al. Systemic reovirus therapy of metastatic cancer in immune-competent mice. Cancer Res 2003; 63: 348-353.

23 Lun XQ, Jang JH, Tang N, Deng H, Head R, Bell JC et al. Efficacy of systemically administered oncolytic vaccinia virotherapy for malignant gliomas is enhanced by combination therapy with rapamycin or cyclophosphamide. Clin Cancer Res 2009; 15: $2777-2788$.

24 Friedman A, Tian JP, Fulci G, Chiocca EA, Wang J. Glioma virotherapy: effects of innate immune suppression and increased viral replication capacity. Cancer Res 2006; 66: 2314-2319.

25 Fulci G, Breymann L, Gianni D, Kurozomi K, Rhee SS, Yu J et al. Cyclophosphamide enhances glioma virotherapy by inhibiting innate immune responses. Proc Natl Acad Sci USA 2006; 103: 12873-12878.

26 Halloran PF. Immunosuppressive drugs for kidney transplantation. N Engl J Med 2004; 351: 2715-2729.

27 Gummert JF, Ikonen T, Morris RE. Newer immunosuppressive drugs: a review. J Am Soc Nephrol 1999; 10: 1366-1380.

28 Allison AC. Mechanisms of action of mycophenolate mofetil. Lupus 2005; 14 (Suppl 1): s2-s8. 
29 Whiteside TL. The tumor microenvironment and its role in promoting tumor growth. Oncogene 2008; 27: 5904-5912.

30 Zou W. Regulatory T cells, tumour immunity and immunotherapy. Nat Rev Immunol 2006; 6: 295-307.

31 Gabrilovich DI, Nagaraj S. Myeloid-derived suppressor cells as regulators of the immune system. Nat Rev Immunol 2009; 9: 162-174.

32 Coffelt SB, Hughes R, Lewis CE. Tumor-associated macrophages: effectors of angiogenesis and tumor progression. Biochim Biophys Acta 2009; 1796: 11-18.

33 Lewis CE, Pollard JW. Distinct role of macrophages in different tumor microenvironments. Cancer Res 2006; 66: 605-612.

34 Mantovani A, Sozzani S, Locati M, Allavena P, Sica A. Macrophage polarization: tumor-associated macrophages as a paradigm for polarized M2 mononuclear phagocytes. Trends Immunol 2002; 23: 549-555.

35 Breitbach CJ, Paterson JM, Lemay CG, Falls TJ, McGuire A, Parato KA et al. Targeted inflammation during oncolytic virus therapy severely compromises tumor blood flow. Mol Ther 2007; 15: $1686-1693$

36 Kurozumi K, Hardcastle J, Thakur R, Yang M, Christoforidis G, Fulci $\mathrm{G}$ et al. Effect of tumor microenvironment modulation on the efficacy of oncolytic virus therapy. J Natl Cancer Inst 2007; 99: 1768-1781.

37 Prestwich RJ, Errington F, Diaz RM, Pandha HS, Harrington KJ, Melcher AA et al. The case of oncolytic viruses versus the immune system: waiting on the judgment of Solomon. Hum Gene Ther 2009; 20: 1119-1132.

38 Munguia A, Ota T, Miest T, Russell SJ. Cell carriers to deliver oncolytic viruses to sites of myeloma tumor growth. Gene Therapy 2008; 15: 797-806.

39 Power AT, Bell JC. Taming the Trojan horse: optimizing dynamic carrier cell/oncolytic virus systems for cancer biotherapy. Gene Therapy 2008; 15: 772-779.

40 Raykov Z, Rommelaere J. Potential of tumour cells for delivering oncolytic viruses. Gene Therapy 2008; 15: 704-710.

41 Hamada K, Desaki J, Nakagawa K, Zhang T, Shirakawa T, Gotoh A et al. Carrier cell-mediated delivery of a replication- competent adenovirus for cancer gene therapy. Mol Ther 2007; 15: 1121-1128.

42 Iankov ID, Blechacz B, Liu C, Schmeckpeper JD, Tarara JE, Federspiel MJ et al. Infected cell carriers: a new strategy for systemic delivery of oncolytic measles viruses in cancer virotherapy. Mol Ther 2007; 15: 114-122.

43 Power AT, Wang J, Falls TJ, Paterson JM, Parato KA, Lichty BD et al. Carrier cell-based delivery of an oncolytic virus circumvents antiviral immunity. Mol Ther 2007; 15: 123-130.

44 Ilett EJ, Prestwich RJ, Kottke T, Errington F, Thompson JM, Harrington KJ et al. Dendritic cells and T cells deliver oncolytic reovirus for tumour killing despite pre-existing anti-viral immunity. Gene Therapy 2009; 16: 689-699.

45 Ichihashi Y. Extracellular enveloped vaccinia virus escapes neutralization. Virology 1996; 217: 478-485.

46 Ojalvo LS, King W, Cox D, Pollard JW. High-density gene expression analysis of tumor-associated macrophages from mouse mammary tumors. Am J Pathol 2009; 174: 1048-1064.

47 Willmon C, Harrington K, Kottke T, Prestwich R, Melcher A, Vile R. Cell carriers for oncolytic viruses: Fed Ex for cancer therapy. Mol Ther 2009; 17: 1667-1676.

48 Lamfers ML, Fulci G, Gianni D, Tang Y, Kurozumi K, Kaur B et al. Cyclophosphamide increases transgene expression mediated by an oncolytic adenovirus in glioma-bearing mice monitored by bioluminescence imaging. Mol Ther 2006; 14: 779-788.

49 Altomonte J, Wu L, Chen L, Meseck M, Ebert O, Garcia-Sastre A et al. Exponential enhancement of oncolytic vesicular stomatitis virus potency by vector-mediated suppression of inflammatory responses in vivo. Mol Ther 2008; 16: 146-153.

$50 \mathrm{Li}$ Q, Bartlett DL, Gorry MC, O'Malley ME, Guo ZS. Three epigenetic drugs up-regulate homeobox gene Rhox 5 in cancer cells through overlapping and distinct molecular mechanisms. Mol Pharmacol 2009; 76: 1072-1081.

51 Zhang X, Goncalves R, Mosser DM. The isolation and characterization of murine macrophages. Curr Protoc Immunol 2008; Chapter 14: Unit: 14.1.2-14.1.4.

52 Mosser DM, Zhang X. Activation of murine macrophages. Curr Protoc Immunol 2008; Chapter 14: Unit 4: 14.4.1-14.4.3. 